Wang Li-Hua, Liu Li-Li, Cao Shu-Hua and Tai Xi-Shi*

\title{
Crystal structure of poly[ $\left(\mathrm{m}_{3}-3-\right.$ \\ carboxyadamantane-1-carboxylato- $\left.\mathrm{K}^{3} 0: 0^{\prime}: 0^{\prime \prime}\right)$ - (phenanthroline- $\left.\mathrm{K}^{2} N, N^{\prime}\right)$ sodium(II)], $\mathrm{C}_{24} \mathrm{H}_{23} \mathrm{~N}_{2} \mathrm{NaO}_{4}$
}

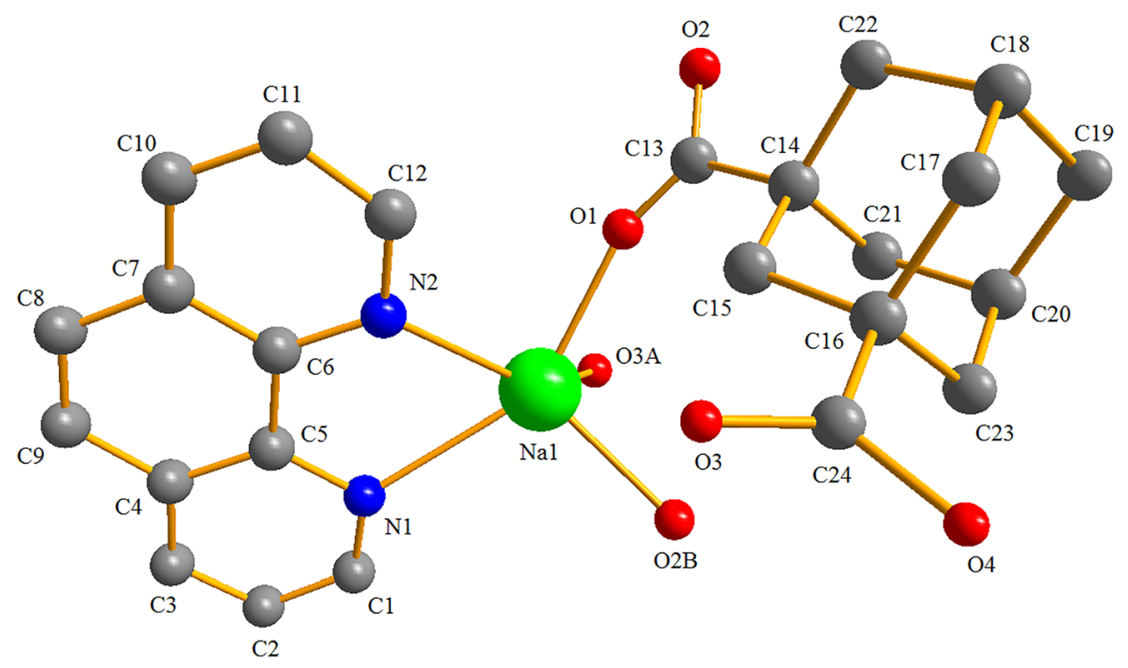

https://doi.org/10.1515/ncrs-2021-0067

Received February 19, 2021; accepted March 11, 2021;

published online March 30, 2021

\section{Abstract}

$\mathrm{C}_{24} \mathrm{H}_{23} \mathrm{~N}_{2} \mathrm{NaO}_{4}$, monoclinic, $P 2_{1} / c$ (no. 14), $a=7.5246(5) \AA$, $b=22.554(2) \AA, c=11.3358(9) \AA, \beta=91.881(7)^{\circ}, V=1922.8(3) \AA^{3}$, $Z=4, R_{g t}(F)=0.0496, w R_{\text {ref }}\left(F^{2}\right)=0.1184, \mathrm{~T}=100 \mathrm{~K}$.

\section{CCDC no.: 1974244}

A part of the title coordination polymer is shown in the figure. Table 1 contains crystallographic data and Table 2 contains the list of the atoms including atomic coordinates and displacement parameters.
Table 1: Data collection and handling.

\begin{tabular}{|c|c|}
\hline Crystal: & Colourless block \\
\hline Size: & $0.12 \times 0.11 \times 0.09 \mathrm{~mm}$ \\
\hline Wavelength: & Mo $K \alpha$ radiation $(0.71073 \AA$ ) \\
\hline$\mu$ : & $0.12 \mathrm{~mm}^{-1}$ \\
\hline Diffractometer, scan mode: & SuperNova, $\omega$ \\
\hline$\theta_{\max }$, completeness: & $25.0^{\circ},>99 \%$ \\
\hline$N(h k l)_{\text {measured }}, N(h k l)_{\text {unique }}, R_{\text {int }}:$ & $8172,3372,0.046$ \\
\hline Criterion for $I_{\mathrm{obs}}, N\left(h k l_{\mathrm{gt}}\right.$ : & $I_{\text {obs }}>2 \sigma\left(I_{\text {obs }}\right), 2495$ \\
\hline$N(\text { param })_{\text {refined }}:$ & 281 \\
\hline Programs: & $\begin{array}{l}\text { Bruker [1], Olex2 [2], SHELX [3], } \\
\text { Diamond [4] }\end{array}$ \\
\hline
\end{tabular}

\section{Source of material}

The mixture of $0.112 \mathrm{~g}$ 1,3-adamantanedicarboxylic acid (0.5 mmol), $0.040 \mathrm{~g} \mathrm{NaOH}(1.0 \mathrm{mmol})$ were dissolved in $15 \mathrm{~mL}$ water-ethanol $(v: v=2: 1)$ at room temperature. After $0.5 \mathrm{~h}, 0.090 \mathrm{~g}$ 1,10-phenanthroline monohydrate $(0.5 \mathrm{mmol})$ was added to the aforementioned solution and stirred for $6 \mathrm{~h}$ at $65{ }^{\circ} \mathrm{C}$. Then the reaction mixture was cooled to room temperature and filtered. The colorless crystals of the title compound were obtained from the filtrate in 30 days.
*Corresponding author: Tai Xi-Shi, College of Chemistry and Chemical Engineering, Weifang University, Weifang, Shandong 261061, P. R. China, E-mail: taixs@wfu.edu.cn. https://orcid.org/0000-00020050-1900

Wang Li-Hua, Liu Li-Li and Cao Shu-Hua, College of Chemistry and Chemical Engineering, Weifang University, Weifang, Shandong 261061, P. R. China 
Table 2: Fractional atomic coordinates and isotropic or equivalent isotropic displacement parameters $\left(\AA^{2}\right)$.

\begin{tabular}{|c|c|c|c|c|}
\hline Atom & $x$ & $y$ & $z$ & $U_{\text {iso }} * U_{\text {eq }}$ \\
\hline $\mathrm{C} 1$ & $0.0022(3)$ & $0.45503(11)$ & $0.8129(2)$ & $0.0165(6)$ \\
\hline $\mathrm{H} 1$ & -0.031624 & 0.423859 & 0.860911 & $0.020^{*}$ \\
\hline $\mathrm{C} 2$ & $-0.0378(3)$ & $0.51265(11)$ & $0.8489(2)$ & $0.0173(6)$ \\
\hline $\mathrm{H} 2$ & -0.096983 & 0.519198 & 0.918494 & $0.021^{*}$ \\
\hline $\mathrm{C} 3$ & $0.0118(3)$ & $0.55947(11)$ & $0.7796(2)$ & $0.0166(6)$ \\
\hline H3 & -0.012781 & 0.598253 & 0.801698 & 0.020 * \\
\hline $\mathrm{C} 4$ & $0.1005(3)$ & $0.54772(11)$ & $0.6746(2)$ & $0.0133(5)$ \\
\hline $\mathrm{C} 5$ & $0.1345(3)$ & $0.48799(10)$ & $0.6455(2)$ & $0.0118(5)$ \\
\hline $\mathrm{C} 6$ & $0.2261(3)$ & $0.47479(11)$ & $0.5372(2)$ & $0.0121(5)$ \\
\hline $\mathrm{C} 7$ & $0.2768(3)$ & $0.52253(10)$ & $0.4648(2)$ & $0.0127(5)$ \\
\hline $\mathrm{C} 8$ & $0.2377(3)$ & $0.58205(11)$ & $0.4968(2)$ & $0.0163(6)$ \\
\hline $\mathrm{H} 8$ & 0.271284 & 0.612867 & 0.447742 & $0.020^{*}$ \\
\hline $\mathrm{C} 9$ & $0.1524(3)$ & $0.59471(11)$ & $0.5975(2)$ & $0.0148(6)$ \\
\hline H9 & 0.127540 & 0.633881 & 0.616582 & $0.018^{*}$ \\
\hline $\mathrm{C} 10$ & $0.3714(3)$ & $0.50889(11)$ & $0.3636(2)$ & $0.0152(6)$ \\
\hline $\mathrm{H} 10$ & 0.408424 & 0.539044 & 0.314099 & $0.018^{\star}$ \\
\hline $\mathrm{C} 11$ & $0.4087(3)$ & $0.45110(11)$ & $0.3380(2)$ & $0.0166(6)$ \\
\hline $\mathrm{H} 11$ & 0.472778 & 0.441376 & 0.272007 & $0.020^{*}$ \\
\hline $\mathrm{C} 12$ & $0.3484(3)$ & $0.40693(11)$ & $0.4133(2)$ & $0.0174(6)$ \\
\hline $\mathrm{H} 12$ & 0.372202 & 0.367714 & 0.393986 & $0.021^{*}$ \\
\hline $\mathrm{C} 13$ & $0.2139(3)$ & $0.24483(10)$ & $0.3746(2)$ & $0.0125(5)$ \\
\hline C14 & $0.3716(3)$ & $0.20694(10)$ & $0.41933(19)$ & $0.0105(5)$ \\
\hline $\mathrm{C} 15$ & $0.5033(3)$ & $0.24358(10)$ & $0.49773(19)$ & $0.0111(5)$ \\
\hline $\mathrm{H} 15 \mathrm{~A}$ & 0.554629 & 0.274868 & 0.451035 & $0.013^{*}$ \\
\hline H15B & 0.440048 & 0.261888 & 0.561425 & $0.013^{*}$ \\
\hline $\mathrm{C} 16$ & $0.6526(3)$ & $0.20373(10)$ & $0.5496(2)$ & $0.0114(5)$ \\
\hline $\mathrm{C} 17$ & $0.7526(3)$ & $0.17564(11)$ & $0.4463(2)$ & $0.0152(6)$ \\
\hline $\mathrm{H} 17 \mathrm{~A}$ & 0.805742 & 0.206615 & 0.399705 & $0.018^{\star}$ \\
\hline $\mathrm{H} 17 \mathrm{~B}$ & 0.847213 & 0.150278 & 0.477331 & $0.018^{*}$ \\
\hline $\mathrm{C} 18$ & $0.6240(3)$ & $0.13925(11)$ & $0.3683(2)$ & $0.0182(6)$ \\
\hline $\mathrm{H} 18$ & 0.688359 & 0.121997 & 0.302838 & $0.022^{*}$ \\
\hline C19 & $0.5440(3)$ & $0.08962(11)$ & $0.4412(2)$ & $0.0229(6)$ \\
\hline $\mathrm{H} 19 \mathrm{~A}$ & 0.637621 & 0.063862 & 0.472153 & $0.027^{\star}$ \\
\hline H19B & 0.463331 & 6074 & 0.391885 & $0.027^{\star}$ \\
\hline $\mathrm{C} 20$ & $0.4430(3)$ & $0.11696(11)$ & $0.5434(2)$ & $0.0203(6)$ \\
\hline $\mathrm{H} 2 \mathrm{O}$ & 0.391887 & 0.085130 & 0.590431 & $0.024^{\star}$ \\
\hline $\mathrm{C} 21$ & $0.2930(3)$ & $0.15709(10)$ & $0.4952(2)$ & $0.0154(6)$ \\
\hline $\mathrm{H} 21 \mathrm{~A}$ & 0.229978 & 0.174279 & 0.560227 & 0.019 * \\
\hline $\mathrm{H} 21 \mathrm{~B}$ & 0.209066 & 0.133866 & 0.447693 & $0.019^{*}$ \\
\hline $\mathrm{C} 22$ & $0.4731(3)$ & $0.17870(11)$ & $0.3190(2)$ & $0.0158(6)$ \\
\hline $\mathrm{H} 22 \mathrm{~A}$ & 0.522098 & 0.209571 & 0.270156 & $0.019^{*}$ \\
\hline $\mathrm{H} 22 \mathrm{~B}$ & 0.392137 & 0.155096 & 0.270047 & 0.019 * \\
\hline $\mathrm{C} 23$ & $0.5706(3)$ & $0.15382(11)$ & $0.6225(2)$ & $0.0155(6)$ \\
\hline $\mathrm{H} 23 \mathrm{~A}$ & 0.664051 & 0.128552 & 0.655317 & $0.019^{*}$ \\
\hline $\mathrm{H} 23 \mathrm{~B}$ & 0.506481 & 0.170723 & 0.687252 & $0.019^{*}$ \\
\hline $\mathrm{C} 24$ & $0.7908(3)$ & 0.23927 (11) & 0.62139 (19) & $0.0124(5)$ \\
\hline $\mathrm{N} 1$ & $0.0857(2)$ & $0.44171(9)$ & $0.71437(17)$ & $0.0141(5)$ \\
\hline $\mathrm{N} 2$ & $0.2594(2)$ & $0.41705(9)$ & $0.51083(17)$ & $0.0136(5)$ \\
\hline $\mathrm{Na} 1$ & $0.10141(11)$ & $0.33590(4)$ & $0.61115(8)$ & $0.0155(3)$ \\
\hline 01 & $0.15148(19)$ & $0.28324(7)$ & $0.43964(14)$ & $0.0174(4)$ \\
\hline 02 & $0.1487(2)$ & $0.23262(7)$ & $0.27031(13)$ & $0.0171(4)$ \\
\hline 03 & 81203 (19) & $0.29281(7)$ & $0.60832(14)$ & $0.0167(4)$ \\
\hline 04 & $0.8911(2)$ & $0.20685(7)$ & $0.69295(14)$ & $0.0178(4)$ \\
\hline H4 & 0.975163 & 0.226742 & 0.718311 & $0.027^{\star}$ \\
\hline
\end{tabular}

\section{Experimental details}

The hydrogen atoms were positioned geometrically $(\mathrm{C}-\mathrm{H}=0.93-0.97 \AA$ and $\mathrm{O}-\mathrm{H}=0.82 \AA)$. Their $U_{\text {iso }}$ values were set to $1.2 U_{e q}$ or $1.5 U_{e q}$ of the parent atoms.

\section{Comment}

Carboxylate metal complexes have shown excellent properties in many aspects such as catalytic properties, luminescence and magnetic properties, as well as cytotoxic activities [5-7]. Therefore, it is of great significance to continue to study the structure and properties of carboxylate metal complexes. In our previous work, some carboxylate metal complexes have been synthesized, and their structures have also been characterized [8-11]. In order to study the structures of carboxylate metal complexes, a new $\mathrm{Na}(\mathrm{I})$ carboxylate coordination polymer has been synthesized and structurally characterized.

Single-crystal X-ray diffraction reveals that the $\mathrm{Na}(\mathrm{I})$ coordination polymer features a 2D framework. The asymmetric unit contains one sodium(I) ion, one phenanthroline (phen) and one 3-carboxyadamantane-1-carboxylate anion (ADA). The $\mathrm{Na}(\mathrm{I})$ center is five coordinated with three carboxylate oxygen atoms (01, O3A, O2B) from three different mono deprotonated ADA ligands and two nitrogen atoms (N1, N2) from one phen ligand. The $\mathrm{Na}-\mathrm{O}$ and $\mathrm{Na}-\mathrm{N}$ distances are in the range of 2.3197(18)-2.662(2) $\AA$, which are comparable to other $\mathrm{Na}(\mathrm{I})$ coordination polymers. All ADA ligands possess the same coordination mode, e.g., one deprotonated carboxylate group bridges two different $\mathrm{Na}(\mathrm{I})$ ions and the other carboxylate group coordinates with another $\mathrm{Na}(\mathrm{I})$ ion [12]. The carboxylic oxygen atoms bridge the $\mathrm{Na}(\mathrm{I})$ ions to generate infinite $1 \mathrm{D}$ chains. These chains are further linked by the uncoordinated oxygen atoms to form an extended 2D layer. As a result, the 2D layers form a 3D supramolecular framework by the interaction of $\pi-\pi$ stacking interactions between the phen molecules and the weak hydrogen bonding interactions.

Author contributions: All the authors have accepted responsibility for the entire content of this submitted manuscript and approved submission.

Research funding: National Natural Science Foundation of China (No. 21171132), the Natural Science Foundation of Shandong (ZR2014BL003), the project of Shandong Province Higher Educational Science and Technology Program (J14LC01) and Science Foundation of Weifang. 
Conflict of interest statement: The authors declare no conflicts of interest regarding this article.

\section{References}

1. Bruker. SAINT and SADABS; Bruker AXS Inc.: Madison, Wisconsin, USA, 2000

2. Dolomanov O. V., Bourhis L. J., Gildea R. J., Howard J. A. K., Puschmann H. OLEX2: a complete structure solution, refinement and analysis program. J. Appl. Crystallogr. 2009, 42, 339-341.

3. Sheldrick G. M. Crystal structure refinement with SHELXL. Acta Crystallogr. 2015, C71, 3-8.

4. Brandenburg K. DIAMOND. Visual Crystal Structure Information System. Ver. 3.2; Crystal Impact: Bonn, Germany, 2012.

5. Malinowski J., Sikorski A., Jacewicz D., Drzeżdżon J. A new complex compound of chromium(III) with 5-aminopyridine-2-carboxylate anions- structure, physicochemical and catalytic properties. Polyhedron 2020, 186, 114616.

6. Du J.-Q., Dong J.-L., Xie F., Yang R.-X., Lan H.-M., Wang D.-Z. Lanthanide complexes supported via benzimidazole carboxylic acid ligand: synthesis, luminescence and magnetic properties. J. Mol. Struct. 2020, 1202, 127345.
7. Lovison D., Allegri L., Baldan F., Ballico M., Damante G., Jandl C., Baratta W. Cationic carboxylate and thioacetate ruthenium(II) complexes: synthesis and cytotoxic activity against anaplastic thyroid cancer cells. Dalton Trans 2020, 49, 8375-8388.

8. Tai X.-S., Zhou X.-J., Liu L.-L. Synthesis, crystal structure and antitumor activity of a $\mathrm{Na}(\mathrm{I})$ coordination polymer based on 2-propyl-4,5-imidazoledicarboxylic acid and 1,10-phenanthroline ligands. Chin. J. Struct. Chem. 2019, 38, 1079-1085.

9. Tai X.-S., Guo H.-M., Guo Q.-Q. Synthesis, crystal structure and antitumor activity of a novel $\mathrm{Zn}$ (II) complex with 2(nicotinoyloxy)acetic acid ligand. Chin. J. Struct. Chem. 2018, 37, 1052-1056.

10. Tai X.-S., Jiang G.-Y., Yu Y.-T., Yao H., Wang A.-J. Bis(ethanol-кO)bis(6-aminopicolinato- $\kappa^{2} N, O$ )manganese(II), $\mathrm{C}_{16} \mathrm{H}_{22} \mathrm{O}_{6} \mathrm{~N}_{4} \mathrm{Mg}$. Z. Kristallogr. NCS 2019, 234, 289-290.

11. Tai X.-S., Zhou X.-J., Liu L.-L., Cao S.-H., Wang L.-H. The crystal structure of diaqua-bis(6-aminopicolinato- $\left.\kappa^{2} N, O\right)$ manganese(II), $\mathrm{C}_{12} \mathrm{H}_{14} \mathrm{O}_{6} \mathrm{~N}_{4} \mathrm{Mg}$. Z. Kristallogr. NCS 2020, 235, 605-606.

12. Nie Cong-Kui, Zhao Dan, Tian Ye, Crystal structure of poly[aqua$\left(\mu_{5}-2,5\right.$-dicarboxybenzoato-k $\left.\mathrm{K}^{5} \mathrm{O}: 0: O^{\prime}: O^{\prime \prime}: O^{\prime \prime \prime}\right)$ sodium(I)], $\mathrm{C}_{9} \mathrm{H}_{7} \mathrm{NaO}_{7}$. Z. Kristallogr. NCS 2018, 233, 103-104. 